\title{
AC 2012-3162: DOES EXPLAINING EQUATIONS IN THEIR OWN WORDS HELP STUDENTS ATTACH PHYSICAL MEANING TO THE EQUATIONS?
}

Dr. Marie Lopez del Puerto, University of Saint Thomas

Marie Lopez del Puerto completed her B.S. in physics at Universidad de las Americas, Puebla, in Puebla, Mexico, and her Ph.D. in physics at the University of Minnesota, Twin Cities in Minneapolis, Minn. She is currently an Assistant Professor in the Physics Department at the University of Saint Thomas in St. Paul, Minn. Her research interests include the structural, optical and electronic properties of nanoscale systems, computational physics, and physics and engineering education.

Dr. Jeff Jalkio, University of Saint Thomas

Dr. Paul R. Ohmann, University of Saint Thomas

Paul R. Ohmann received a B.A. from the University of St. Thomas in St. Paul, Minn., and an M.S. and Ph.D. in physics from the University of Wisconsin, Madison. He is currently an Associate Professor in the Physics Department at the University of St. Thomas in St. Paul, Minn. His current interests involve computational physics and complex systems, as well as physics and engineering education. 


\title{
Does explaining equations in their own words help students attach physical meaning to the equations?
}

\begin{abstract}
In order for students to correctly solve a given problem, they must have a good understanding of the different physical situations described by the equations in their toolbox. This means that students must associate equations with their physical meaning and not just see equations as collections of letters and mathematical symbols into which to plug numbers. This past fall semester, we asked students in two undergraduate Physics courses to explain important equations in their own words in a variety of pedagogical ways. To motivate and test their understanding, exams and quizzes that students took during the semester included an open-ended "Explain this equation in words" question, which we scored based on the depth of understanding shown. There are a few questions we are interested in exploring: As students practice this skill, do their explanations become better (i.e. do individual students show deeper understanding as the semester progresses)? Is a student's score in the "Explain this equation in words" question on each exam correlated to his/her total exam score and to his/her final grade? Does the emphasis of putting equations into words result in greater conceptual understanding? We will use standardized pre- and post-tests given in each of our courses to see if there is a difference in gain as compared to past semesters. We will present data from three sections of our second-semester calculus-based Physics course, and from our first-semester junior-level Electricity and Magnetism course.
\end{abstract}

\section{Introduction}

The study we present in this paper arose from two separate trends in our university and the larger higher-education community, plus an observation. The first is the trend in Physics education to focus on teaching for conceptual understanding, for example, through Peer Instruction ${ }^{1}$ or Workshop Physics (which is part of The Physics Suite). ${ }^{2}$ The second trend is the push to improve student writing through programs such as Writing Across the Curriculum, and in particular through Writing to Learn, where short writing exercises are used to help students think through a concept or a problem. ${ }^{3}$ The observation was that a considerable fraction of our students, while talking to other students in class or asking the instructor a question, was referring to variables in equations by the name of the symbol instead of the physical quantity represented by the symbol; for example a student might say, "the change in potential is the same because the $E$ is the same." It occurred to us that students might be doing this because they were looking at equations as mathematical constructs only and not as an expression of physical relationships or laws. Attaching a physical meaning to equations affects the way we view equations, Redish says, "first, through seeing equations as relations, not as calculational methods, and second, through 'filtering the equation through the physics'.,"

In a typical Physics class, we introduce equations in lecture by motivating them from an example problem and building intuitive relationships. Then we either derive or write out the equation, maybe do an example, and finally students answer conceptual questions and/or solve problems. What is missing is the piece where students reflect on the meaning of the equations. Redish ${ }^{4}$ suggests that we need to "find activities that help our students build knowledge into 
intuitions/understanding." This is what we are trying to achieve through having students write equations in their own words. Our hypothesis is that by giving the students continuous practice in writing they will gain conceptual understanding that will be reflected in their problem solving.

\section{Methodology}

We are conducting this study in three sections of Classical Physics II, the second semester calculus-based introductory physics course, and in one section of the upper-level Electricity and Magnetism. Classical Physics II is taught in a modified workshop style, with lecture, laboratory, and problem-solving combined into a two and a half hour class period. The students in the course are predominantly Electrical and Mechanical Engineering majors, but it is also a required course for Chemistry, Biology, Geology, and Physics majors. Electricity and Magnetism (E\&M), on the other hand, is lecture based, although in the last two years the instructor has added an optional problem-solving session that is well attended by students. $E \& M$ is almost exclusively taken by Electrical Engineering and Physics majors.

We first introduced "Equations in Words" in our courses as part of homework or in-class problem packets. We showed students an example of an equation in words to clarify the expectation that they were not to do a literal translation symbol by symbol but that they had to think about the physical meaning of the equation. A typical "Equations in Words" problem from the Waves unit in Classical Physics II is shown below:

Consider the following equation:

$$
\Delta \mathrm{L}=(n+1 / 2) \lambda \quad n=0,1,2,3, \ldots
$$

a) This equation applies to:

b) The variables and their units are:
$\Delta \mathrm{L}$
$n$
$\lambda$

c) Explain the equation in your own words:

d) Draw a diagram of a physical situation where this equation would apply. Label all important quantities.

In Classical Physics II, we included an "Equation in Words" question in each of the six unit exams, while in $E \& M$ the "Equation in Words" question was part of a quiz given the class period prior to each of the four unit exams. 
A typical test/quiz question:

Explain in your own words the following equation: $\vec{B}=\frac{\mu_{0}}{4 \pi} \int \frac{I d \vec{l} \times \hat{r}}{r^{2}}$

We scored the student responses using a 5-point scale:

0 : no answer

1: wrong or incomplete answer

2: literal answer, symbols identified by correct physical quantities

3: shows some understanding of physical meaning

4: shows deep understanding of physical meaning

The following are examples of student responses scored according to the scale above:

1. A wrong or incomplete answer: "The magnetic field is proportional to the current, radius constants, and change in length."

2. A literal answer: "The magnetic field $(\vec{B})$ is equal to the constant $\mu_{0}$ over $4 \pi$ multiplied by the integral of the current $(I)$ times displacement vector of length $(d l)$ crossed the direction vector $(\widehat{r})$ all over $r^{2} . "$

3. A response that shows some physical understanding: "This equation says that a magnetic field is dependent upon the current, the length of the current, and the distance from the current."

4. A response that shows deep physical understanding: "This equation is for the magnetic field generated by an electric current. The magnetic field depends on the magnitude, direction, length, and proximity of the current. $\overrightarrow{d l}$ is a small part of the wire and the unit vector $\hat{r}$ is the direction from $\overrightarrow{d l}$ to a point."

\section{Results}

(1) As students practice writing equations in the own words, do their explanations become better?

In other words, do individual students show deeper understanding as the semester progresses? We were surprised to find that the average "Equation in Words" scores for the different sections do not show an increase throughout the semester for either PHYS 112 or PHYS 341. Perhaps this is because students weren't motivated sufficiently: even though these "Equations in Words" were graded, they nevertheless contributed quite little to the overall course grade.

(2) Is a student's score in the "Explain this equation in words" question on each exam correlated to his/her total exam score and to his/her final grade?

We looked at correlations between the scores students obtained in the "Equations in Words" responses and their exam scores. We did not find a strong correlation between individual exams 
and corresponding "Equations in Words" responses. However, we did find a significant correlation between the average exam and average "Equation in Words" scores. We found a similar correlation between the final grades and "Equations in Words" scores, which makes sense since the final grade is based largely on exam average. Correlations for the different sections are shown in Table 1 and Fig. 1.

\begin{tabular}{|c|c|c|}
\hline & Correlation & Correlation \\
\hline Section & Exam Average & Final Grade \\
\hline PHYS 112-01 & 0.62 & 0.67 \\
\hline PHYS 112-02 & 0.88 & 0.85 \\
\hline PHYS 112-03 & 0.79 & 0.74 \\
\hline PHYS 341-01 & 0.74 & 0.74 \\
\hline
\end{tabular}

Table 1 Correlation between average exam scores and average "Equations in Words" scores, and between final grades and average "Equations in Words" scores, for each course section that participated in this study

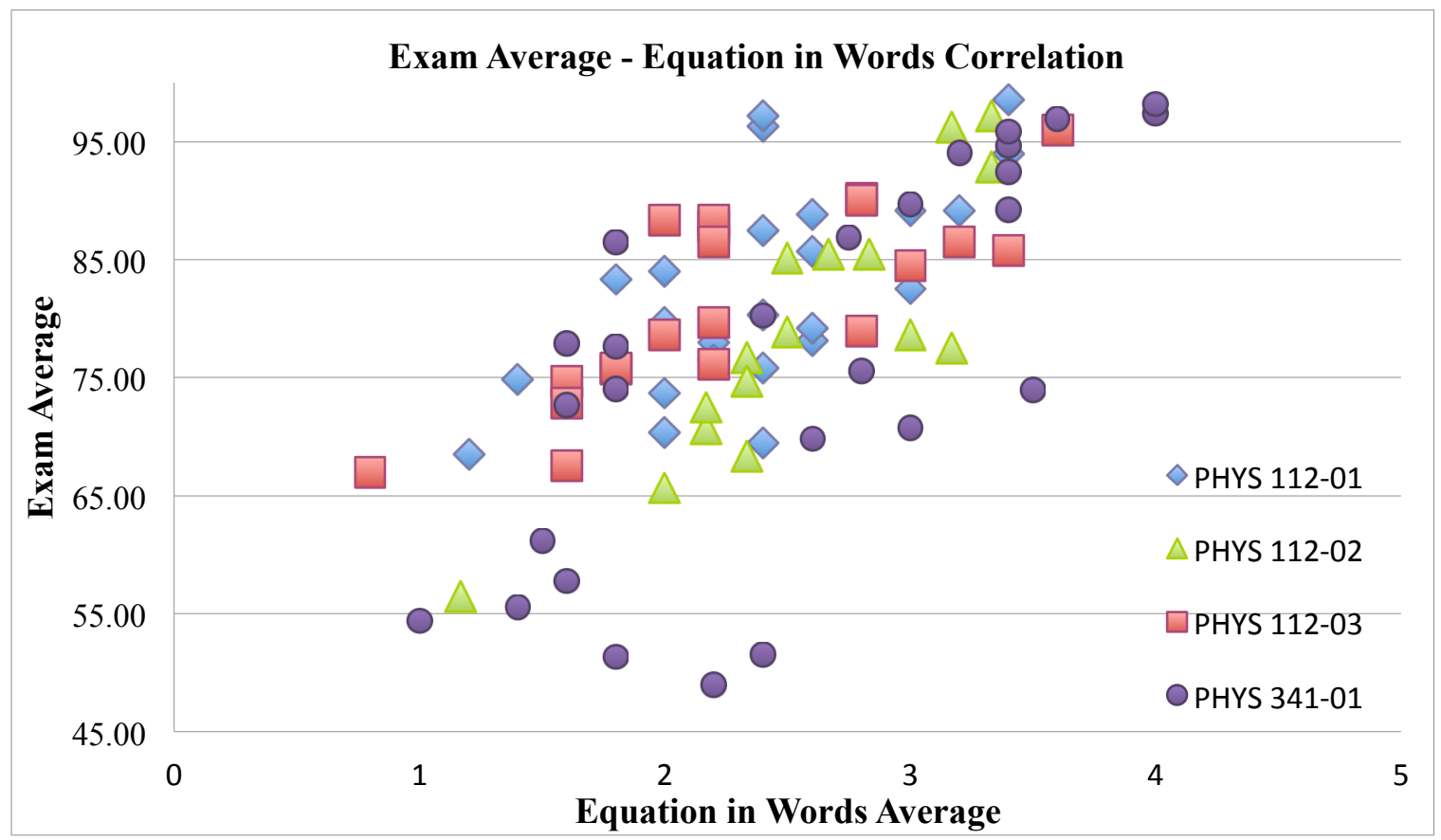

Fig. 1 Correlation between average exam scores and average "Equations in Words" scores.

(3) Does the emphasis of putting equations into words result in greater conceptual understanding?

We looked at correlations between the scores students obtained in the "Equations in Words" and their score in the Brief Electricity and Magnetism Assessment (BEMA) given at the end of the semester. ${ }^{6}$ We found small, but somewhat variable positive correlations, as shown in Table 2. 


\begin{tabular}{|c|c|}
\hline Section & BEMA post-test \\
\hline PHYS 112-01 & 0.64 \\
\hline PHYS 112-02 & 0.22 \\
\hline PHYS 112-03 & 0.16 \\
\hline PHYS 341-01 & 0.61 \\
\hline
\end{tabular}

Table 2 Correlation between BEMA post-test and average "Equations in Words" scores for each course section that participated in this study

We also looked at BEMA normalized gains and how they compare to other semesters. For PHYS 112 , we see quite a bit of fluctuation in BEMA normalized gains from semester to semester. This semester's scores were right on average, so we cannot say whether using "Equations in Words" resulted in a greater conceptual understanding based on the BEMA results.

\section{Conclusion}

This paper presents preliminary data and analysis on a study of the relationship between having students explain equations in writing using their own words, and conceptual understanding of physics. We found that there is a significant correlation between average exam scores and demonstrating physical understanding in the "Equations in Words" questions on the exams, which is not surprising - students who do better on exams should have a better understanding of the material.

We did not see an increase in normalized gains for the BEMA and found small correlations between the BEMA and the average "Equations in Words" score for each student. While we hoped that having "Equations in Words" exercises in group packets and questions in exams would lead students to think more about the meaning of equations, responses to "Equations in Words" questions did not show consistent improvement over time. Apparently, the amount of practice, feedback, and exam points that students got in "Equations in Words" questions was not enough to convince students that this was a worthwhile task that would increase their conceptual understanding and their ability to solve problems. However, the questions included in grouppackets did lead to useful in-class discussions on the different variables, their units, and the relationship between them. "Equation in Words" in-class exercises and quizzes were also useful as feedback to the instructors.

If we were to extend this study in the future and attempt to improve the results, we would need to emphasize the "Equations in Words" to an even greater degree. For example, perhaps increasing the number and weighting of these questions on exams would be beneficial, or perhaps additional class time working with equation definitions would be advantageous. Of course, such emphasis comes with an expense: in our classes, this would reduce the time spent working on traditional problems. In the end, we are most comfortable with the "middle approach" described in this paper, as a balance that emphasizes the meaning of the equations we derive with the applications that use them. 


\section{References}

${ }^{1}$ Eric Mazur, Peer Instruction: A User's Manual, Benjamin Cummings (1996).

${ }^{2}$ Priscilla W. Laws, Workshop Physics, $2^{\text {nd }}$ edition, Wiley (2004).

${ }^{3}$ See for example: Charles Bazerman et al., Reference Guide to Writing Across the Curriculum, Parlor Press (2005); Susan H. McLeod and Margot Soven, Writing across the curriculum: a guide to developing programs, Sage Publications (1992); and Anne J. Herrington, "Writing to Learn: Writing across the Disciplines," College English, V. 43, No. 4, 379 (1981).

${ }^{4}$ Edward F. Redish, "Problem Solving and the use of Math in Physics Courses," conference proceedings, World View on Physics Education in 2005: Focusing on Change, Delhi (2005).

${ }^{5}$ J. Tuminaro, Ph.D. Dissertation, University of Maryland, Physics Department (2004).

${ }^{6}$ Ding, L., Chabay, R., Sherwood, B., \& Beichner, R., "Evaluating an electricity and magnetism assessment tool: Brief electricity and magnetism assessment," Phys. Rev. ST Phys. Educ. Res. 2, pp. 010105-1-7 (2006). 